This item was submitted to Loughborough's Research Repository by the author.

Items in Figshare are protected by copyright, with all rights reserved, unless otherwise indicated.

\title{
Explicit model predictive control on the air path of turbocharged diesel engines
}

\section{PLEASE CITE THE PUBLISHED VERSION}

https://doi.org/10.1109/ACC.2013.6580649

\section{PUBLISHER}

IEEE (@ American Automatic Control Council)

\section{VERSION}

VoR (Version of Record)

\section{PUBLISHER STATEMENT}

This work is made available according to the conditions of the Creative Commons Attribution-NonCommercialNoDerivatives 4.0 International (CC BY-NC-ND 4.0) licence. Full details of this licence are available at: https://creativecommons.org/licenses/by-nc-nd/4.0/

\section{LICENCE}

CC BY-NC-ND 4.0

\section{REPOSITORY RECORD}

Zhao, Dezong, Cunjia Liu, Richard Stobart, Jiamei Deng, and Edward Winward. 2017. "Explicit Model Predictive Control on the Air Path of Turbocharged Diesel Engines". figshare. https://hdl.handle.net/2134/26519. 


\title{
Explicit Model Predictive Control on the Air Path of Turbocharged Diesel Engines
}

\author{
Dezong Zhao, Member, IEEE, Cunjia Liu, Richard Stobart, Member, IEEE, \\ Jiamei Deng, Senior Member, IEEE, and Edward Winward
}

\begin{abstract}
The turbocharged diesel engine is a typical multiinput multi-output (MIMO) system with strong couplings, actuator constraints, and fast dynamics. This paper addresses the air path regulation in turbocharged diesel engines using an explicit model predictive control (EMPC) approach, which allows tracking of the time-varying setpoint values generated by the supervisory level controller while satisfying the actuator constraints. The proposed EMPC framework consists of calibration, engine model identification, controller formulation, and state observer design. The proposed EMPC approach has a low computation requirement and is suitable for implementation in the engine control unit (ECU) on board. The experimental results on a turbocharged Cat ${ }^{\mathbb{B}}$ C6.6 diesel engine illustrate that the EMPC controller significantly improves the tracking performance of the exhaust emission variables against the decentralized single-input single-output (SISO) control method.
\end{abstract}

Index Terms-Explicit model predictive control, turbocharged diesel engines, exhaust emissions regulation

\section{NOMENCLATURE}

$\begin{array}{ll}N & \text { Engine speed. } \\ W_{f} & \text { Engine fueling rate. } \\ W_{c} & \text { Compressor air mass flow rate. } \\ W_{e g r} & \text { EGR mass flow rate. } \\ W_{e} & \text { Engine total mass flow rate. } \\ W_{t} & \text { Turbine gas mass flow rate. } \\ P_{c} & \text { Compressor power. } \\ P_{t} & \text { Turbine power. } \\ p_{\text {in }} & \text { Intake manifold pressure. } \\ p_{\text {exh }} & \text { Exhaust manifold pressure. } \\ p_{a} & \text { Ambient pressure. } \\ V_{i n} & \text { Intake manifold volume. } \\ V_{e x h} & \text { Exhaust manifold volume. } \\ T_{i n} & \text { Intake manifold temperature. } \\ T_{e x h} & \text { Exhaust manifold temperature. } \\ T_{a} & \text { Ambient temperature. } \\ F_{1} & \text { Burnt gas fraction. } \\ N_{t c} & \text { Turbocharger shaft rotating speed. } \\ \lambda_{a} & \text { Air-fuel ratio. } \\ \chi_{e g r} & \text { EGR valve opening percentage. } \\ \chi_{v g t} & \text { VGT vane opening percentage. }\end{array}$

This work was supported by grant funding from the UK's innovation agency, the Technology Strategy Board, through its Collaborative Research and Development Programme.

D. Zhao, C. Liu, R. Stobart, and E. Winward are with Department of Aeronautical and Automotive Engineering, Loughborough University, Loughborough, Leicester, UK (e-mail: d.zhao2@lboro.ac.uk, c.liu5@lboro.ac.uk, r.k.stobart@lboro.ac.uk, r.e.t.b.winward@lboro.ac.uk).

J. Deng is with School of Mechanical and Automotive Engineering, Kingston University, Kingston upon Thames, Surrey, UK (e-mail: j.deng@kingston.ac.uk).

$\begin{array}{ll}\eta_{m} & \text { Turbocharger mechanical efficiency. } \\ R_{g} & \text { Specific gas constant. } \\ \tau & \text { Turbocharger time constant. } \\ n_{c} & \text { Compressor isentropic efficiency. } \\ n_{t} & \text { Turbine isentropic efficiency. } \\ c_{p} & \text { Specific heat at constant pressure. } \\ \gamma & \text { Specific heat ratio, } 1.4 \text { for air. } \\ \mu & \frac{\gamma-1}{\gamma} .\end{array}$

\section{INTRODUCTION}

As the increasingly tighter pollution standards are obliged on the vehicles with internal combustion engines (ICE), more exhaust gas regulation systems are equipped on the diesel engines. On a higher level, developing more effective control strategies on diesel engines is now essential for improving the exhaust emissions [1]. Modern diesel engines are normally equipped with a variable geometry turbocharger (VGT) and exhaust gas recirculation (EGR) valves [2], [3]. Turbocharging the diesel engine reduces fuel consumption, together with the EGR valves enable a reduction in exhaust emissions, in particular nitrogen oxides $\left(\mathrm{NO}_{x}\right)$ [4]. VGT and EGR actuators are strongly coupled because they are all driven by the exhaust gas. They should be well tuned for regulating the intake mass flow for combustion with the desired burnt gas fraction $F_{1}$ to minimize $\mathrm{NO}_{x}$, without violating the air-fuel ratio $\lambda_{a}$ associated with the particulate matter (PM) generation. Unfortunately, the performance variables, $F_{1}$ and $\lambda_{a}$, are unmeasurable using normal sensors. As a consequence, two intermediate variables, $W_{c}$ and $p_{i n}$ are introduced as the new controlled variables, which are closely related with the previous ones [5]. The regulation of $F_{1}$ and $\lambda_{a}$ is correspondingly converted to control $W_{c}$ and $p_{\text {in }}$, which are measurable but exhibit nonlinear dynamics due to the coupling between VGT and EGR actuators.

Most of the commercial ECUs use SISO proportionalintegral-differential (PID) controllers in diesel engine air path control, where one regulates the $W_{c}$ by tuning $\chi_{v g t}$, while another regulates the $p_{\text {in }}$ by tuning $\chi_{\text {egr }}$. However, with the increasingly stricter emission standards, it is more difficult for the decentralized SISO methods to meet the standards without consideration of coupling between the actuators [6]. Therefore, developing control algorithms that can deal with nonlinear dynamics is required. For the capability of handling the constraints on manipulated variables in MIMO systems, model predictive control (MPC) is one of the most promising control strategies in industrial applications [7], [8]. However, realtime implementation of MPC brings high computation burden, 


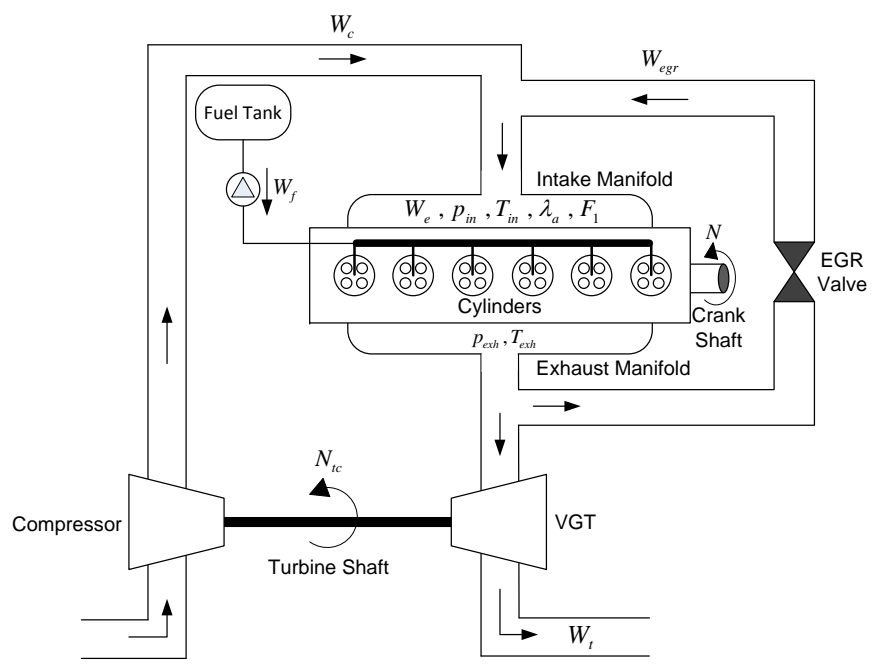

Fig. 1. Turbocharged diesel engine

due to a finite horizon optimal control problem is solved in each sampling period [9]. Higher computation burden brings higher requriements on the processing power of ECUs, and furthermore, higher requirements on the cost. Therefore, high computational cost control methods are improper for production diesel engines. Recently, the explicit MPC (EMPC) has attracted interest in engineering with the potential ability in reducing hardware cost and online computation time [10], [11]. In the EMPC approach, the optimal control laws under different conditions are pre-computed and called from a lookup table, resulting in computational resource requirements are reduced.

In this paper, several aspects in establishing an EMPC framework on diesel engines are proposed. The contributions of this paper mainly focus on the complete procedure in formulating an EMPC controller in exhaust emissions regulation according to the development experience, particularly on the general method in obtaining the multi-linear diesel engine model and building an augmented EMPC controller. Experimental results support the proposed method.

The paper is organized as following. After the introduction in section I, the diesel engine model is described in section II. The EMPC control framework is formulated in section III. The experiment results are stated in section IV. Finally, the conclusions are summarized in section V.

\section{System Description}

The schematic of a turbocharged diesel engine is demonstrated in Fig. 1. The turbocharger consists of the VGT and compressor, where the VGT takes energy from the exhaust gas to spin up the compressor which is mounted on the same turbine shaft, in turns compresses more fresh air, resulting in higher pressure in the intake manifold. The EGR loop feeds back part of the burnt exhaust gas to the intake manifold to dilute the fresh air, causing lower combustion peak temperature and further lower $\mathrm{NO}_{x}$ concentration. The mixed air and burnt gas is pumped into the cylinders from the intake manifold. As the piston reaches the top of its compression stroke, fuel is

injected into the cylinders from the fuel tank and burnt with the pumped air, producing torque on the crank shaft. The hot burnt exhaust gas is pumped into the exhaust manifold from the cylinders, where part of the exhaust gas flows out of the engine through the VGT, and the other part is recirculated back to the intake manifold through the EGR valves.

Since EGR valves and VGT vane are both driven by the exhaust gas, there is a strong coupling between the EGR flow and VGT flow. The reduction of the pumped fresh air in the intake manifold leads to an increase of PM emissions while a low value of EGR flow fraction results in higher $\mathrm{NO}_{x}$ emissions. The dilemma is known as the $\mathrm{NO}_{x}$-PM tradeoff.

\section{A. Diesel Engine Air Path}

The exhaust performance variables of the diesel engine are defined as $\mathrm{NO}_{x}$ and $\mathrm{PM}$, while the reduction of them is achieved by keeping a sufficient large value of $F_{1}$ and $\lambda_{a}$, respectively. Therefore, $F_{1}$ and $\lambda_{a}$ are employed as the engine performance variables, which are defined by

$$
F_{1}=\frac{W_{e g r}}{W_{c}+W_{e g r}} \quad \lambda_{a}=\frac{W_{c}}{W_{f}} .
$$

Precise tracking of $F_{1}$ and $\lambda_{a}$ to their optimal setpoint values $F_{1}^{*}$ and $\lambda_{a}^{*}$ is desired, where $F_{1}^{*}$ and $\lambda_{a}^{*}$ can be obtained using a supervisory controller. In conventional environments, $W_{c}$ and $p_{\text {in }}$ are measured by the compressor air flow sensor and the boost pressure sensor, respectively, to provide the information about the intake gas process. $W_{c}$ and $p_{i n}$ have strong coupling and are regulated by tuning both of the VGT vane and EGR valves.

Ignoring the slow deviation of $T_{\text {in }}$ and $T_{\text {exh }}$, a third-order nonlinear control-oriented air path model is formulated with respect to $p_{i n}, p_{\text {exh }}$, and $P_{c}$ :

$$
\begin{aligned}
\dot{p}_{\text {in }} & =\frac{R_{g} T_{\text {in }}}{V_{\text {in }}}\left(W_{c}+W_{\text {egr }}-W_{e}\right), \\
\dot{p}_{\text {exh }} & =\frac{R_{g} T_{\text {exh }}}{V_{\text {exh }}}\left(W_{e}-W_{e g r}-W_{t}+W_{f}\right), \\
\dot{P}_{c} & =\frac{1}{\tau}\left(\eta_{m} P_{t}-P_{c}\right) .
\end{aligned}
$$

$W_{c}$ is related to $P_{c}$ with

$$
W_{c}=\frac{n_{c}}{c_{p} T_{a}} \frac{P_{c}}{p_{i n}^{\mu}-1},
$$

while $P_{t}$ can be expressed by $W_{t}$ :

$$
P_{t}=n_{t} c_{p} T_{e x h}\left(1-p_{e x h}^{\mu}\right) W_{t} .
$$

The mass flow rate through the EGR valve can be obtained by the actuator map given by:

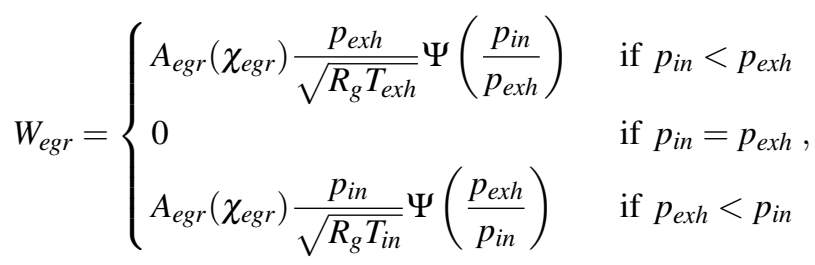


where

$$
\Psi\left(\frac{p_{i}}{p_{j}}\right)=\left\{\begin{array}{c}
\gamma^{0.5}\left(\frac{2}{\gamma+1}\right)^{(\gamma+1) /(2(\gamma-1))} \\
\text { if } \frac{p_{i}}{p_{j}} \leq\left(\frac{2}{\gamma+1}\right)^{\gamma /(\gamma-1)} \\
\sqrt{\frac{2 \gamma}{\gamma-1}\left(\left(\frac{p_{i}}{p_{j}}\right)^{2 / \gamma}-\left(\frac{p_{i}}{p_{j}}\right)^{(\gamma+1) / \gamma}\right)} \\
\text { if } \frac{p_{i}}{p_{j}}>\left(\frac{2}{\gamma+1}\right)^{\gamma /(\gamma-1)}
\end{array}\right.
$$

and $A_{\text {egr }}$ is expressed as a quadratic function with respect to $\chi_{\text {egr }}$. The turbine mass flow rate is represented by a modified version of the orifice equation:

$$
W_{t}=A_{v g t}\left(\chi_{v g t}\right) \frac{p_{\text {exh }}}{\sqrt{R T_{\text {exh }}}} \Phi\left(\frac{p_{a}}{p_{\text {exh }}}, \chi_{v g t}\right),
$$

where $A_{v g t}$ is a quadratic function with respect to $\chi_{v g t}$ and $\Phi\left(\frac{p_{a}}{p_{\text {exh }}}, \chi_{\mathrm{vgt}}\right)$ is obtained from a VGT mass flow rate map. The reader can refer to [2] for further details on the air path dynamics.

\section{B. Linearized Model}

Generally, $W_{f}$ reveals the influence of the load torque $T_{L}$ on the engine. At an engine determined operation point with fixed $N$ and $T_{L}$, the diesel engine air path can be modeled as a linear system in form of discrete state space equations:

$$
\left\{\begin{array}{l}
x(k+1)=A x(k)+B u(k) \\
y(k)=C x(k)
\end{array},\right.
$$

where $x \in \mathbb{R}^{n}$ is the state vector, $u \in \mathbb{R}^{m}$ is the input vector, $y \in \mathbb{R}^{p}$ is the output vector, and $(A, B)$ is a controllable pair. Both inputs and outputs of the system are constrained with respect to their maximum and minimum bounds:

$$
u_{\min } \leq u \leq u_{\max }, \quad y_{\min } \leq y \leq y_{\max } .
$$

The coefficient matrices $A, B$, and $C$ are obtained via system identification at the selected operation point. The inputs and outputs of the linearized model for the air path are selected as

$$
\begin{aligned}
& u=\left[\begin{array}{ll}
\chi_{e g r} & \chi_{v g t}
\end{array}\right]^{\mathrm{T}}, \\
& y=\left[\begin{array}{ll}
W_{c} & p_{i n}
\end{array}\right]^{\mathrm{T}} .
\end{aligned}
$$

According to different engine speed and load torque, the engine operation region is segregated into several subzones. The number of zones depends on the precision of the required identified model. In each subzone, a linear model is identified at the geometrical central point.

\section{EMPC FRAMEWORK DESIGN}

As shown in Fig. 2, the implementation of EMPC can be divided into two stages: offline and online. In the offline stage, the diesel engine model is identified using the calibration data. Based on the identified model, the EMPC control laws are calculated via multi-parametric quadratic programming method and are stored in the look-up table within the ECU.
In the online stage, the diesel engine is controlled by the precomputed control law, and the feedback states are estimated via the state observer, fulfilling the closed-loop control function.

\section{A. Calibration and System Identification}

Considering the nonlinear behavior of the diesel engine, it is infeasible to obtain a unified linear model in the engine operation range. A more practical approach is to identify the piecewise affine models in smaller operation ranges.

The calibration data set should cover the operation range of the diesel engine to be tested. System identification is implemented based on the calibration data which are separated into two parts: training data and validation data. A group of candidate models with different orders should be generated from the training data. The one with the highest fitting score in validation data is selected as the proper linear model in the assigned zone. Generally speaking, the air path model is ranging from $2^{\text {nd }}$ to $4^{\text {th }}$ order.

\section{B. EMPC Controller Design}

The EMPC controller described in this subsection adopts the linear MPC technique to achieve tracking of the given output variables.

1) Problem Formulation: The setpoints of output variables are incorporated into the standard EMPC formulation for tracking of time varying reference values. An augmented formulation of (8) including the input dynamics $u(k)=u(k-1)+$ $\Delta u(k)$ and the setpoints of outputs is represented as:

$$
\left\{\begin{array}{c}
\underbrace{\left[\begin{array}{c}
x(k+1) \\
u(k) \\
r(k+1)
\end{array}\right]}_{\tilde{x}_{k+1}}=\underbrace{\left[\begin{array}{ccc}
A & B & 0 \\
0 & I & 0 \\
0 & 0 & I
\end{array}\right]}_{\tilde{A}} \underbrace{\left[\begin{array}{c}
x(k) \\
u(k-1) \\
r(k)
\end{array}\right]}_{\tilde{y}_{k}}+\underbrace{\left[\begin{array}{c}
B \\
I \\
0
\end{array}\right]}_{\tilde{B}} \underbrace{\Delta u(k)}_{\tilde{x}_{k}},
\end{array}\right.
$$

where $\tilde{x}_{k} \in \mathbb{R}^{n+m+p}, \tilde{u}_{k} \in \mathbb{R}^{m}, \tilde{y}_{k} \in \mathbb{R}^{p}$, with $\tilde{C}=[C, 0,-I]$; $r(k)$ is the desired setpoint of $y(k)$.

For a generalized system, the system performance index with the initial state $\tilde{x}_{k}$ at time instant $k$ can be specified by a

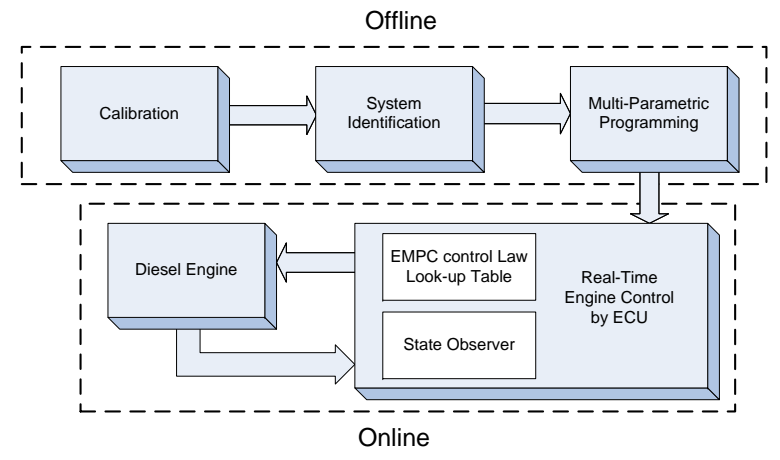

Fig. 2. Implementation procedure of EMPC on the diesel engine 
quadratic cost function to be minimized:

$$
\begin{array}{ll}
\min _{U} & J\left(\tilde{x}_{k}, \tilde{U}\right)=\underbrace{\left\|\tilde{x}_{k+H_{p}}\right\|_{P}^{2}}_{J\left(\tilde{x}_{k+H_{p}}\right)}+\sum_{i=0}^{H_{p}-1} \underbrace{\left(\left\|\tilde{x}_{k+i}\right\|_{Q}^{2}+\left\|\tilde{u}_{k+i}\right\|_{R}^{2}\right)}_{J\left(\tilde{x}_{k+i}, \tilde{u}_{k+i}\right)}, \\
\text { s.t. } & \tilde{x}_{k+i+1}=\tilde{A} \tilde{x}_{k+i}+\tilde{B} \tilde{u}_{k+i} \\
& \tilde{u}_{\min } \leq \tilde{u}_{k+i} \leq \tilde{u}_{\max }, \quad i=0,1, \ldots, H_{c}-1 \\
& \tilde{u}_{k+i}=\tilde{u}_{k+H_{c}-1}, \quad i=H_{c}, \ldots, H_{p} \\
& \tilde{y}_{\min } \leq \tilde{y}_{k+i} \leq \tilde{y}_{\max }, \quad i=1, \ldots, H_{p}
\end{array}
$$

where $\|x\|_{S}^{2}=x^{\mathrm{T}} S x ; H_{p}$ and $H_{c}$ are the prediction horizon and control horizon, respectively; $\tilde{x}_{k+i}$ and $\tilde{y}_{k+i}$ denote the predictions of $\tilde{x}$ and $\tilde{y}$ at time $k+i$, made at time $k$, respectively; $\tilde{u}_{k+i}$ is the value of control input $\tilde{u}$ at time $k+i ; \tilde{U}=\left[\tilde{u}_{k}^{\mathrm{T}}, \ldots, \tilde{u}_{k+H_{p}-1}\right]^{\mathrm{T}} \in \mathbb{R}^{m H_{p}}$ is the control sequence within which the optimal control inputs be determined; $\tilde{X}=$ $\left[\tilde{x}_{k+1}^{\mathrm{T}}, \cdots, \tilde{x}_{H_{p}}^{\mathrm{T}}\right]^{\mathrm{T}} \in \mathbb{R}^{(n+m+p) H_{p}}$ is the vector of predicted states; $J\left(\tilde{x}_{k+H_{p}}\right)$ is the terminal penalty function, while $J\left(\tilde{x}_{k+i}, \tilde{u}_{k+i}\right)$ is the stage cost at time $k+i$; From physical point of view, $H_{c} \leq H_{p}$ should be guaranteed. $P=P^{\mathrm{T}}>0, Q=Q^{\mathrm{T}}>0$ and $R=R^{\mathrm{T}}>0$ are assumed.

2) Multi-Parametric Quadratic Programming: The optimization problem (11) can be converted into a quadratic programming (QP) formation for on which fast and numerically reliable algorithms are available. Rewrite the cost function (11a) in the following quadratic form:

$$
J\left(\tilde{x}_{k}, \tilde{U}\right)=\tilde{X} \bar{Q} \tilde{X}+\tilde{U} \bar{R} \tilde{U},
$$

where the augmented matrices $\bar{Q}$ and $\bar{R}$ are $\bar{Q}=$ $\operatorname{diag}(Q, \cdots, Q, P)$ and $\bar{R}=\operatorname{diag}(R, \cdots, R)$, respectively.

For each element $\tilde{x}_{k+i}$, the evolution of the system (10), i.e. the equality constraint (11b), can be represented by

$$
\tilde{x}_{k+i}=\tilde{A}^{i} \tilde{x}_{k}+\sum_{j=0}^{i-1} \tilde{A}^{j} \tilde{B} \tilde{u}_{k+i-1-j},
$$

for $i=1,2, \ldots, H_{p}$, which means the system states at any time can be expressed in terms of the initial state $\tilde{x}_{k}$ and the inputs vector $\tilde{U}$. Therefore, a matrix expression of the system states evolution can be derived from (13):

$$
\tilde{X}=\bar{A} \tilde{x}_{k}+\bar{B} \tilde{U},
$$

with

$$
\bar{A}=\left[\begin{array}{c}
\tilde{A} \\
\tilde{A}^{2} \\
\vdots \\
\tilde{A}^{H_{c}} \\
\vdots \\
\tilde{A}^{H_{p}}
\end{array}\right] \quad \bar{B}=\left[\begin{array}{cccc}
\tilde{B} & 0 & \cdots & 0 \\
\tilde{A} \tilde{B} & \tilde{B} & \cdots & 0 \\
\vdots & \vdots & \vdots & \vdots \\
\tilde{A}^{H_{c}-1} \tilde{B} & \tilde{A}^{H_{c}-2} \tilde{B} & \cdots & 0 \\
\vdots & \vdots & \vdots & \vdots \\
\tilde{A}^{H_{p}-1} \tilde{B} & \tilde{A}^{H_{p}-2} \tilde{B} & \cdots & \tilde{B}
\end{array}\right] .
$$

Substituting (14) into (12), the optimization problem can be translated into solving the following QP problem :

$$
\begin{aligned}
& J\left(\tilde{x}_{k}, \tilde{U}\right)=\tilde{x}_{k}^{\mathrm{T}} Y \tilde{x}_{k}+\min _{\tilde{U}}\left\{\tilde{U}^{\mathrm{T}} H \tilde{U}+2 \tilde{x}_{k}^{\mathrm{T}} F \tilde{U}\right\}, \\
& \text { s.t. } G \tilde{U} \leq W+E \tilde{x}_{k},
\end{aligned}
$$

where $Y=\bar{A}^{\mathrm{T}} \bar{Q} \bar{A}, H=\bar{B}^{\mathrm{T}} \bar{Q} \bar{B}+\bar{R}$, and $F=\bar{A}^{\mathrm{T}} \bar{Q} \bar{B}$. The matrices $G, W$, and $E$ can be obtained from the constraints (11c) and (11e). The readers can refer to [11] for more details.

Introducing $z \triangleq \tilde{U}+H^{-1} F^{\mathrm{T}} \tilde{x}_{k} \in \mathbb{R}^{m H_{p}}$, the optimization problem (16) can be rewritten as

$$
\begin{gathered}
J_{z}\left(\tilde{x}_{k}, \tilde{U}\right)=\min _{z} z^{\mathrm{T}} H z, \\
\text { s.t. } \quad G z \leq W+S \tilde{x}_{k},
\end{gathered}
$$

where $S \triangleq E+G H^{-1} F^{\mathrm{T}}$, and $J_{z}\left(\tilde{x}_{k}, \tilde{U}\right)=J\left(\tilde{x}_{k}, \tilde{U}\right)-\frac{1}{2} \tilde{x}_{k}^{\mathrm{T}}(Y-$ $\left.F H^{-1} F^{\mathrm{T}}\right) \tilde{x}_{k}$. The QP problem (17) can be solved by applying the Karush-Kuhn-Tucker (KKT) optimality conditions [?]:

$$
\begin{aligned}
H z+G^{\mathrm{T}} & =0, \\
\lambda_{i}\left(G_{i} z-W_{i}-S_{i} \tilde{x}_{k}\right) & =0, \quad i=1, \ldots, q, \\
\lambda & \geq 0 \\
G z-W-S \tilde{x}_{k} & \leq 0
\end{aligned}
$$

where $\lambda \in \mathbb{R}^{q}$ denotes the Lagrange multipliers, and $q$ is the number of inequalities in (17b). The subscript $i$ denotes the $i$-th row of the corresponding matrix.

Let $z^{*}\left(\tilde{x}_{k}\right)$ be the optimal solution to (17) for a given $\tilde{x}_{k}$, the constraint (18b) is called active if $G_{i} z^{*}\left(\tilde{x}_{k}\right)-W_{i}-S_{i} \tilde{x}_{k}=0$ is held. Accordingly, the matrices on the corresponding rows are denoted as $\tilde{G}, \tilde{W}$, and $\tilde{S}$, respectively. Substituting (18b) into (18a), the active Lagrange multiplier $\tilde{\lambda}$ is solved as:

$$
\tilde{\lambda}=-\left(\tilde{G} H^{-1} \tilde{G}^{\mathrm{T}}\right)^{-1}\left(\tilde{W}+\tilde{S} \tilde{x}_{k}\right) .
$$

Substituting (19) into (18a) yields

$$
z=H^{-1} \tilde{G}^{\mathrm{T}}\left(\tilde{G} H^{-1} \tilde{G}^{\mathrm{T}}\right)^{-1}\left(\tilde{W}+\tilde{S} \tilde{x}_{k}\right) .
$$

It is clear from (19) and (20) that $\tilde{\lambda}$ and $z$ are affine functions of $\tilde{x}_{k}$. Substituting (19) and (20) into (18c) and (18d), the region satisfying the constraints can be determined by

$$
\begin{aligned}
-\left(\tilde{G} H^{-1} \tilde{G}^{\mathrm{T}}\right)^{-1}\left(\tilde{W}+\tilde{S} \tilde{x}_{k}\right) & \geq 0, \\
G H^{-1}\left(\tilde{G} H^{-1} \tilde{G}^{\mathrm{T}}\right)^{-1}\left(\tilde{W}+\tilde{S} \tilde{x}_{k}\right) & \leq W+S \tilde{x}_{k} .
\end{aligned}
$$

After removing redundant constraints, (21) describes a polyhedron in the $\tilde{x}_{k}$-space, denoted as the critical region $C R_{0}$, which is represented by

$$
C R_{0}=\left\{\tilde{x}_{k} \mid D \tilde{x}_{k} \leq d, D \in \mathbb{R}^{N_{c} \times(n+m+p)}, d \in \mathbb{R}^{N_{c}}\right\},
$$

where $N_{c}=\operatorname{dim}(d)$ is the total number of inequalities in $D \tilde{x}_{k} \leq d$, which is translated from the constraint (16b). Similar method is also used on dividing the rest of the region:

$$
R_{\text {rest }}=\left\{\tilde{x}_{k} \mid D \tilde{x}_{k} \geq d\right\} .
$$

As shown in [12], the optimal EMPC control law is a continuous piecewise affine function of $\tilde{x}_{k}$ on each divided region:

$$
\tilde{U}^{*}\left(\tilde{x}_{k}\right)=f_{j} \tilde{x}_{k}+g_{j}, \quad j=1, \ldots, N_{j},
$$

where $N_{j}$ is the number of polyhedral sets defined by (22) and (23). 
3) Implementation: In the offline stage, the optimal EMPC control laws are computed explicitly. Once the multiparametric QP problem is solved, the EMPC control law (24) is available explicitly. Only the first component of the vector $\tilde{U}^{*}\left(\tilde{x}_{k}\right)$ be applied:

$$
\tilde{u}_{k}=[I, 0, \ldots, 0] \tilde{U}^{*}\left(\tilde{x}_{k}\right) .
$$

The open-source MPT toolbox based on MATLAB is used to calculate the linear MPC law. On the next step, $\tilde{U}^{*}\left(\tilde{x}_{k+1}\right)$ is searched in the pre-calculated look-up table again, and $\tilde{u}_{k+1}$ is updated accordingly. It shares the same function as the normal MPC method, but needs lower hardware cost and reduced online computation resources. The main advantage of using the EMPC as the engine controller is the ability to handle the control and output constraints, so the exhaust emissions of the diesel engine can be retained in a reasonable range without violating the manipulation mode.

\section{State Estimation}

In this subsection, a Kalman filter is selected for state estimation, which is considered as the the optimal recursive data processing algorithm, and has the highest efficiency in solving most of the engineering problems [13]-[15]. Kalman filter uses the state space equation and recursive method to observe the states, and has no requirements on the smooth or time invariant characteristics of the signal. Without loss of generality, considering the diesel engine disturbed by Gaussian white noises, which are represented by the process error $v(k)$ and the measurement error $w(k)$, the augmented system model (10) is transformed to

$$
\left\{\begin{array}{l}
\tilde{x}_{k+1}=\tilde{A} \tilde{x}_{k}+\tilde{B} \tilde{u}_{k}+v(k) \\
\tilde{y}_{k}=\tilde{C} \tilde{x}_{k}+w(k)
\end{array}\right.
$$

where $v(k)$ and $w(k)$ are independent, and hold the covariance of $Q_{v}$ and $R_{w}$, respectively.

The Kalman filter is composed by two sequential steps: prediction and correction. In the prediction step, the prediction of the states are

$$
\hat{\tilde{x}}_{k+1 \mid k}=\tilde{A} \hat{\tilde{x}}_{k \mid k}+\tilde{B} \tilde{u}_{k}
$$

where $\hat{\tilde{x}}_{k+1 \mid k}$ and $\hat{\tilde{x}}_{k \mid k}$ are the estimates of $\tilde{x}_{k+1}$ and $\tilde{x}_{k}$ by the given output sequence of $\left[\tilde{y}_{k}, \tilde{y}_{k-1}, \cdots\right]$, respectively. In the correction step, the updated estimate of $\tilde{x}_{k+1}$ is obtained by

$$
\hat{\tilde{x}}_{k+1 \mid k+1}=\hat{\tilde{x}}_{k+1 \mid k}+\Delta \tilde{x}_{k+1},
$$

where

$$
\begin{aligned}
\Delta \tilde{x}_{k+1} & =K_{k+1} \Delta \tilde{y}_{k+1}, \\
K_{k+1} & =P_{k+1 \mid k} \tilde{C}\left(\tilde{C} P_{k+1 \mid k} \tilde{C}^{\mathrm{T}}\right)^{-1}, \\
\Delta \tilde{y}_{k+1} & =\tilde{y}_{k+1}-\tilde{C} \hat{\tilde{x}}_{k+1 \mid k}, \\
P_{k+1 \mid k} & =\tilde{A} P_{k \mid k} \tilde{A}^{\mathrm{T}}+Q_{v}, \\
P_{k \mid k} & =E\left(\left(\tilde{x}_{k}-\hat{\tilde{x}}_{k \mid k}\right)\left(\tilde{x}_{k}-\hat{\tilde{x}}_{k \mid k}\right)^{\mathrm{T}}\right) .
\end{aligned}
$$

Finally, $P_{k+1 \mid k+1}$ is updated:

$$
P_{k+1 \mid k+1}=P_{k+1 \mid k}-K_{k+1} \tilde{C} P_{k+1 \mid k} \tilde{C}^{\mathrm{T}} K_{k+1}^{\mathrm{T}} .
$$

In the diesel engine model, the covariance matrices of the process noise and measurement noise are specified as $Q_{v}=\operatorname{diag}\left[Q_{1}, Q_{2}\right]$ and $R_{w}=\operatorname{diag}\left[R_{1}, R_{2}\right]$ in the air path control system, respectively; or $Q_{v}=\operatorname{diag}\left[Q_{1}^{\prime}, Q_{2}^{\prime}, Q_{3}^{\prime}\right]$ and $R_{w}=$ $\operatorname{diag}\left[R_{1}^{\prime}, R_{2}^{\prime}, R_{3}^{\prime}\right]$ in the fuel path control system, respectively.

\section{EXPERIMENTAL RESULTS}

The performance of the proposed control method is evaluated in test-cell experiments. In air path test, the comparison of using the EMPC scheme and the valves-fixed control mode is given.

\section{A. Experiment Setup}

The investigated engine is a Cat ${ }^{R}$ C6.6 ACERT $^{\mathrm{TM}}$ heavyduty off-highway engine. The engine is a 6-cylinder, 6.6-liter engine equipped with a Cat $(\mathbb{R})$ common rail fuel system. The engine calibration used for this work produces up to $159 \mathrm{~kW}$ at rated speed of $2200 \mathrm{rpm}$ with peak torque of $920 \mathrm{Nm}$ occurring at $1400 \mathrm{rpm}$. The engine has been modified with a high pressure loop EGR and a Honeywell servo-actuated twin-stage VGT. The engine is fully instrumented to measure air, fuel and cooling system pressure, temperatures, and flow rates. Emissions data is gathered principally from AVL 415 smoke meter, AVL 439 opacity meter, and a Horiba 9100 exhaust gas analyzer measuring $\mathrm{NO}_{x}, \mathrm{CO}_{x}$, hydrocarbons, and oxygen. The engine is mounted with a Engineering Cadet V14 dynamometer control system coupled to a Froude AG400-HS eddy current dynamometer, which are used to manage the engine speed and load torque.

\section{B. Control Performance Evaluation}

In air path control tests, the command engine speed is 1550 rpm, while the load torque changes from $375 \mathrm{Nm}$ to $475 \mathrm{Nm}$ during a ramping time $t_{\text {ramp }}$. The two selected operation points are both located in a subzone, which means the same identified model are used in control. The sampling period of the ECU is $0.1 \mathrm{~s}$. The setpoint value of $W_{c}$ changes from $8 \mathrm{~kg} / \mathrm{min}$ to 9.5 $\mathrm{kg} / \mathrm{min}$ with the ramping change of the load torque, while the setpoint value of $p_{\text {in }}$ changes from $162 \mathrm{kPa}$ to $190 \mathrm{kPa}$. The constraints on the inputs $u=\left[\begin{array}{ll}\chi_{\text {egr }} & \chi_{v g t}\end{array}\right]^{\mathrm{T}}$ are defined as

$$
u_{\min }=\left[\begin{array}{c}
5 \% \\
45 \%
\end{array}\right], \quad u_{\max }=\left[\begin{array}{l}
15 \% \\
65 \%
\end{array}\right] .
$$

The prediction horizon and control horizon are set as $H_{p}=8$, $H_{c}=2$, respectively.

The air path control performance evaluation with $t_{\text {ramp }}=$ $10 \mathrm{~s}$ is given in Fig. 3. Both of the valves-fixed control mode and the proposed EMPC control method are tested. In the valves-fixed control mode, the air path dynamics behave as an open-loop control system.

Fig. 3(a) and Fig. 3(b) shows a clear improvement of the transient dynamics of the EMPC controller over the fixed control mode. This is because the EMPC is a MIMO control method which considers both the internal plant coupling and actuator constraints. In the fixed control mode, $\chi_{v g t}$ is kept at $55 \%$ by PID tuning, and the $\chi_{\text {egr }}$ is kept at $10 \%$. As a result 


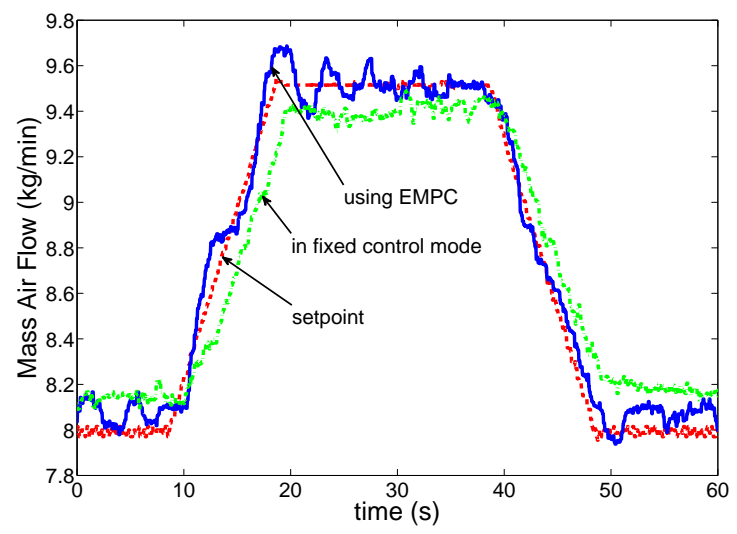

(a) $W_{c}$

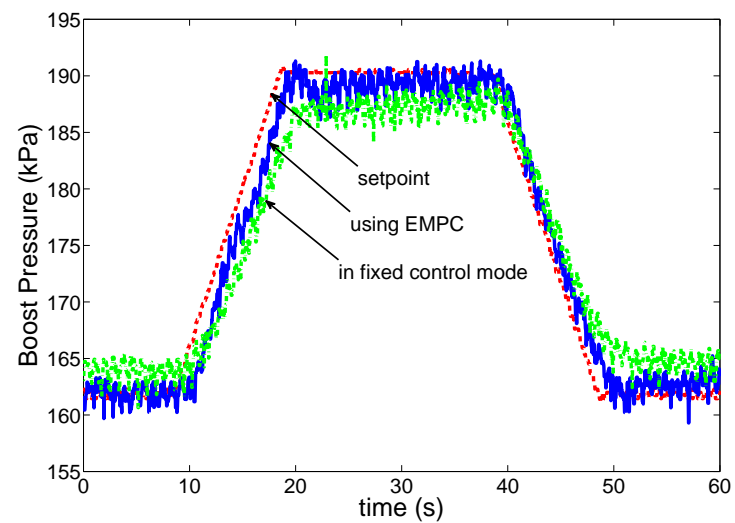

(b) $p_{\text {in }}$
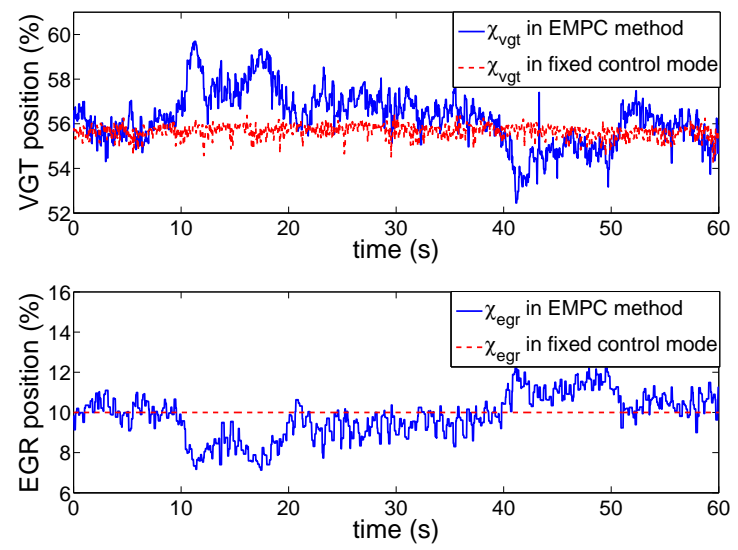

(c) Valves position

Fig. 3. Diesel engine air path control performance evaluation with $t_{\text {ramp }}=$ $10 \mathrm{~s}$

of the open-loop control, the transient performance of the air path is slow and the steady state error is high. It is clear that the tracking performance on the air path is faster and more accurate using the EMPC controller, for the optimal control laws can be selected from pre-calculated solutions quickly.

Fig. 3(c) shows the tuning process of $\chi_{v g t}$ and $\chi_{e g r}$ according to the variations of engine operation points. With the increasing setting values of $W_{c}$ and $p_{i n}, \chi_{v g t}$ increases and $\chi_{e g r}$ decreases, which means more fresh air is boosted and less exhaust gas is recirculated, in order to meet a higher $\lambda_{a}$. With the decreasing setting values of $W_{c}$ and $p_{i n}, \chi_{v g t}$ decreases and $\chi_{\text {egr }}$ increases, since less fresh air and more recirculated exhaust gas are desirable.

\section{CONCLUSIONS}

An EMPC scheme has been proposed on the diesel engine exhaust emissions regulation. Comparing with the traditional MPC, EMPC provides a time-saving way in realtime applications, while maintaining the identical performance as MPC. The state-space models of the diesel engine are identified at several steady operation points, with reasonable hypothesis. An augmented EMPC controller is formulated, and the increment of control actions are considered, such that the tracking is improved and the steady state errors are significantly diminished. Experimental results show that the proposed EMPC strategy holds high precision in trajectories tracking, as well as the high robustness against the step change in the load torque.

\section{REFERENCES}

[1] X. Wang, H. Waschl, D. Alberer, and L. del Re, "A design framework for predictive engine control," Oil \& Gas Science and Technology, vol. 66, no. 4, pp. 599-612, 2011.

[2] M. Jankovic and I. Kolmanovsky, "Constructive Lyapunov control design for turbocharged diesel engines," IEEE Transactions on Control Systems Technology, vol. 8, no. 2, pp. 288-299, 2000.

[3] A. G. Stefanopoulou, I. Kolmanovsky, and J. S. Freudenberg, "Control of variable geometry turbocharged diesel engines for reduced emissions," IEEE Transactions on Control Systems Technology, vol. 8, no. 4, pp. 733-745, 2000.

[4] A. Plianos and R. K. Stobart, "Nonlinear airpath control of modern diesel powertrains: a fuzzy systems approach," International Journal of Systems Science, vol. 42, pp. 263-275, 2011.

[5] S. Garcia-Nieto, M. Martinez, X. Blasco, and J. Sanchis, "Nonlinear predictive control based on local model networks for air management in diesel engines," Control Engineering Practice, vol. 16, no. 12, pp. 1399-1413, 2008.

[6] T. Maruyama, T. Shimura, A. Ejiri, Y. Ikai, and K. Shimotani, "Model predictive control applied to a diesel engine air-path system with dead time," in Proceedings of the SICE Annual Conference, 2011, pp. 26282633.

[7] Y. Zheng, S. Y. Li, and X. B. Wang, "Horizon-varying model predictive control for accelerated and controlled cooling process," IEEE Transactions on Industrial Electronics, vol. 58, no. 1, pp. 329-336, 2011.

[8] F. Barrero, J. Prieto, E. Levi, R. Gregor, S. Toral, M. J. Duran, and M. Jones, "An enhanced predictive current control method for asymmetrical six-phase motor drives," IEEE Transactions on Industrial Electronics, vol. 58, no. 8, pp. 3242-3252, 2011.

[9] H. J. Ferreau, P. Ortner, P. Langthaler, L. del Re, and M. Diehl, "Predictive control of a real-world diesel engine using an extended online active set strategy," Annual Reviews in Control, vol. 31, no. 2, pp. 293-301, 2007.

[10] P. Ortner and L. del Re, "Predictive control of a diesel engine air path," IEEE Transactions on Control Systems Technology, vol. 15, no. 3, pp. 449-456, 2007.

[11] M. Kvasnica, Real-time model predictive control via multi-parametric programming: theory and tools. Saarbrucken, Germany: VDM Verlag Dr. Muller, 2009.

[12] A. Bemporad, M. Morari, V. Dua, and E. N. Pistikopoulos, "The explicit linear quadratic regulator for constrained systems," Automatica, vol. 38, no. 1, pp. 3-20, 2002.

[13] M. Barut, S. Bogosyan, and M. Gokasan, "Speed-sensorless estimation for induction motors using extended Kalman filters," IEEE Transactions on Industrial Electronics, vol. 54, no. 1, pp. 272-280, 2007.

[14] S. H. P. Won, W. W. Melek, and F. Golnaraghi, "A Kalman/particle filter-based position and orientation estimation method using a position sensor/inertial measurement unit hybrid system," IEEE Transactions on Industrial Electronics, vol. 57, no. 5, pp. 1787-1798, 2010.

[15] T. Schuhmann, W. Hofmann, and R. Werner, "Improving operational performance of active magnetic bearings using Kalman filter and state feedback control," IEEE Transactions on Industrial Electronics, vol. 59, no. 2, pp. 821-829, 2012. 